\title{
Exciting the Edge Mode in a Dual Metasurface PTD Symmetric Waveguide
}

\author{
Marzieh SalarRahimi ${ }^{1}$, Guy A. E. Vandenbosch ${ }^{2}$ \\ ${ }^{1}$ ESAT-TELEMIC, KU Leuven, Leuven, Belgium, marzieh.salarrahimi@kuleuven.be \\ ${ }^{2}$ ESAT-TELEMIC, KU Leuven, Leuven, Belgium, guy.vandenbosch@kuleuven.be
}

\begin{abstract}
In this paper, we propose a symmetric dual metasurface-based waveguide. The metasurfaces provide a duality impedance, which can support a linear edge mode at the interface of the metasurfaces. The mode is a very confined TEM mode and is protected against backscattering. The metasurfaces are implemented by a couple of complementary structures. One is a bed of nails and the other is a holey structure.
\end{abstract}

Index Terms-Metamaterials, metasurfaces, topologycal EM, bed of nails, holey structure.

\section{INTRODUCTION}

Recently, the application of topological photonics for manipulating electromagnetic wave propagation and scattering has attracted considerable attention. Topological edge modes support robust unidirectional propagation along an edge, without reflections from structural imperfections [1]. Topological edge mode protection normally requires nonreciprocal elements, which make the system complex. On the other hand, parity time-reversal duality (PTD) symmetric systems, which can be formed only by reciprocal materials, are good alternatives for topological systems [2].

It was proved in [3] that PTD symmetric systems may support waves that are insensitive to any form of perturbation or discontinuity that satisfies the PTD symmetry. A PTD-symmetric guide formed by pairing two dual parallel-plate waveguides (PPWs) with perfect electric conductor (PEC) and perfect magnetic conductor (PMC) walls was proposed in [4]. The fundamental mode of this structure was analytically analyzed in [2]. The mode is strongly confined along the discontinuity of the boundary. Some physical structures also were presented, implementing the proposed waveguide.

In this paper, we propose a PTD-symmetric waveguide formed by pairing a couple of parallel-plate waveguides. Two complementary structures, with transverse-magnetic (TM) or transverse-electric (TE) fundamental modes, are used to make each parallel-plate waveguide. Replacing the PEC-PMC walls in [2] with complementary structures adds more degrees of freedom to the design of PTD-symmetric waveguides.

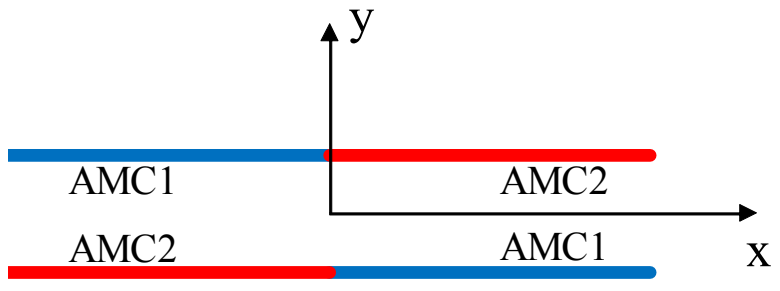

Fig. 1. Conceptual schematic of the proposed dual-AMC PTDsymmetric waveguide.

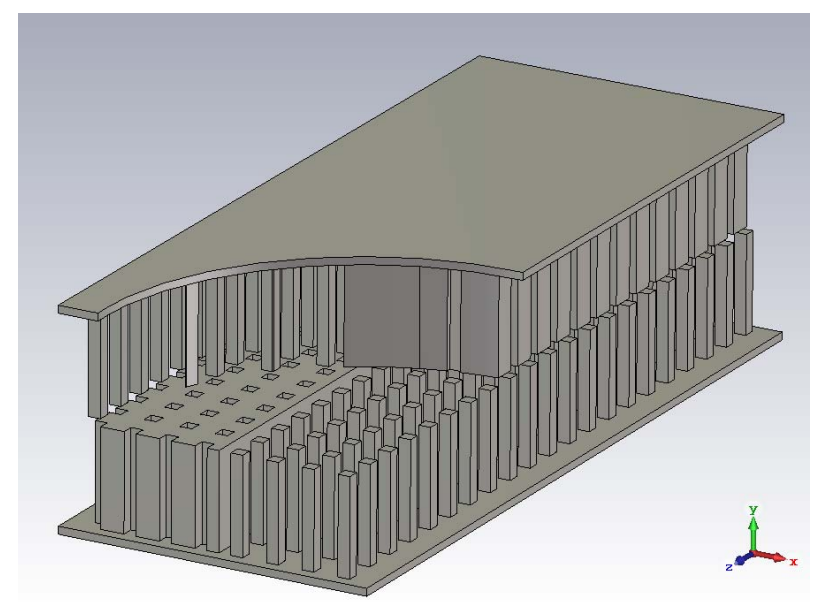

Fig. 2. The proposed dual-AMC PTD-symmetric waveguide.

\section{Edge Mode In Dual Metasurface Parallel Plate WaVEGUIDE}

Fig. 1 shows a conceptual drawing of the proposed PTD-symmetric parallel-plate waveguide. The AMC1 and AMC2 represent metamaterial-based artificial magnetic conductor surfaces. These are implemented by two complementary structures, which support complementary impedances for TM or TE polarized waves. The eigenmode analytic theory in [5] proved the existence of an edge mode, which occurs between the two complementary surface impedances. 


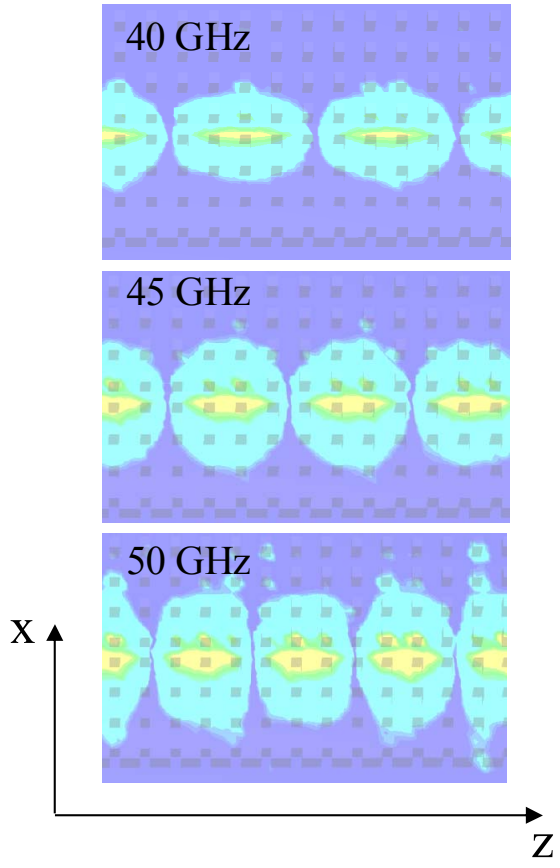

Fig. 3. Field distribution of the TEM edge mode at several frequencies.

\section{Design the Dual Metasurfaces WaVEguide}

In this section, a physical structure is presented to prove the concept. The AMC surfaces are implemented by two complementary structures. As shown in Fig. 2, one of them is a bed of nails and another one is a holey structure. The structure is simulated using CST. Fig. 3 depicts the field distribution of the TEM edge mode at different frequencies. As expected, the wave is strongly confined at the interface of the parallel-plate waveguides. Fig. 4 compares the ability of confining energy at the interface for the proposed dualAMC structure and the PEC-PMC structure in [2]. An improvement of $47 \%$ in the $3 \mathrm{db}$ - field confinement width is achieved. Since the field distribution mostly confined along the edge, this can be interpreted as an edge mode.

In addition, the dispersion properties of the excited TEM-like edge mode are investigated. As Fig. 5 shows, the mode propagates with a group velocity less than the light velocity. Actually, it is a slow wave. Therefore, it not only is confined in the lateral direction but also in its propagation direction. This advantage opens a huge opportunity to design different EM devices based on the proposed topology.

\section{CONCLUSIONS}

In this conference paper, a new PTD symmetric waveguide is introduced. The waveguide supports a strongly confined TEM-like edge mode, which propagates along the interface of two complementary metasurfaces. The concept is implemented by a bed of nails and a holey AMC. The

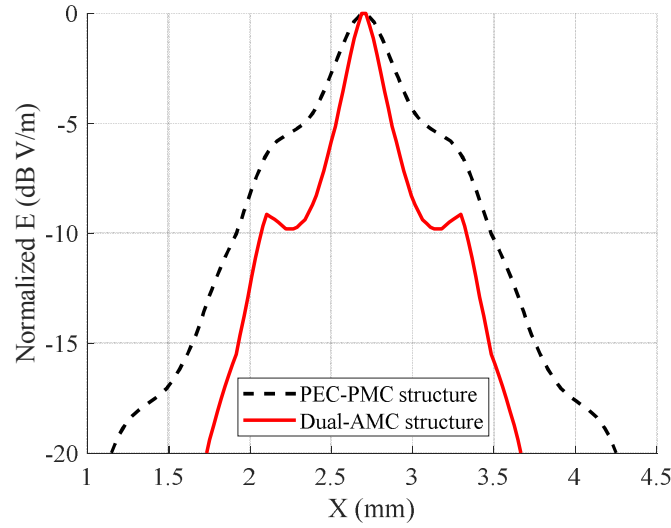

Fig. 4. Field confinement at $40 \mathrm{GHz}$.

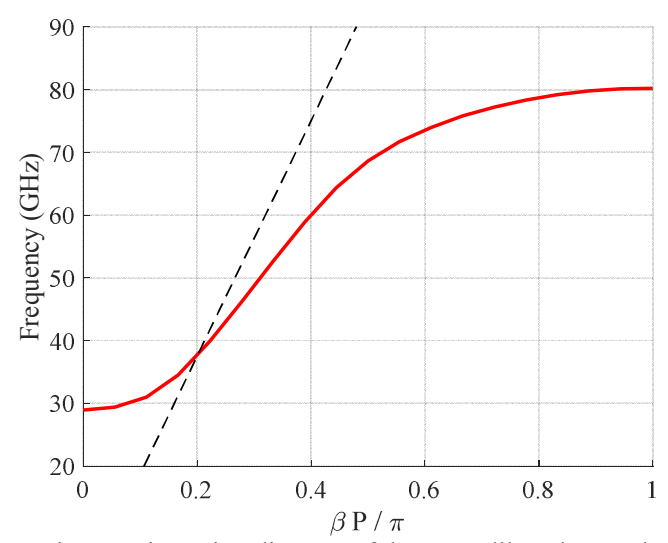

Fig. 5. Dispersion diagram of the TEM-like edge mode.

simulation results show that the proposed structure greatly guides the wave along the edge of the AMC surfaces.

\section{ACKNOWLEDGMENT}

This project has received funding from the European Union's Horizon 2020 research and innovation programme under the Marie Sklodowska-Curie grant agreement No. 721732 .

\section{REFERENCES}

[1] 1 X. Cheng, C. Jouvaud, X. Ni, S. H. Mousavi, A. Z. Genack, and A. B. Khanikaev, Robust reconfigurable electromagnetic pathways within a photonic topological insulator, Nat. Mater. 15, 542 (2016).

[2] 2 E. Martini, M. G. Silveirinha, and S. Maci, Exact solution for the protected TEM edge mode in a PTD-symmetric parallel-plate waveguide, IEEE Trans. Antennas Propag. 67, 1035 (2019).

[3] 3 M. G. Silveirinha, "PTD Symmetry protected scattering anomaly in optics," Phys. Rev. B, vol. 95, 035153, 2017.

[4] 4 W.-J. Chen, Z.-Q. Zhang, J.-W. Dong, and C. T. Chan, "Symmetryprotected transport in a pseudospin-polarized waveguide," Nature Commun., vol. 6, Sep. 2015, Art. no. 8183.

[5] 5 X. Kong, D. J. Bisharat, G. Xiao, and D. F. Sievenpiper, Analytic theory of an edge mode between impedance surfaces, Phys. Rev. A 99, $033842(2019)$ 